\title{
Chemical separation of acrylic color components enabling the identification of the pigment spectroscopic response
}

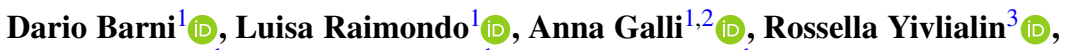 \\ Simone Caglio ${ }^{1}$ (D) Marco Martini $^{1}$ (D), Adele Sassella ${ }^{1, \mathrm{a}}(\mathbb{D})$ \\ ${ }^{1}$ Department of Materials Science, University of Milano - Bicocca, 20125 Milan, Italy \\ 2 CNR-IBFM, Istituto di Bioimmagini e Fisiologia Molecolare - Consiglio Nazionale \\ delle Ricerche, 20090 Segrate, Italy \\ 3 Present Address: Institute for Solar Fuels, Helmholtz-Zentrum Berlin für Materialien und Energie GmbH, \\ Hahn-Meitner-Platz 1, 14109 Berlin, Germany
}

Received: 14 October 2020 / Accepted: 11 February 2021

(C) The Author(s) 2021

\begin{abstract}
Acrylic colors are mixtures of several components that can be identified as pigments, binders, and fillers, so that, when analyzed, the characteristic response of the different components may not be recognizable. This limits the accuracy of spectroscopic techniques, nonetheless particularly useful as they are noninvasive and can be applied in situ on real artworks. Here, a method is proposed to chemically separate and identify the different components of acrylic colors, in order to be able to study their spectroscopic response separately, in particular by ultraviolet-visible-near-infrared diffuse reflectance. The results clearly show that the chemical and analytical method developed here is fully reliable, with the advantage of clearly separating the response of the different components without any change of their chromatic/chemical properties. As a case study, the new method is applied here to original acrylic colors used by the Italian artist Ico Parisi, in view of building a spectra database.
\end{abstract}

\section{Introduction}

The correct conservation and restoration of a painting requires the exact knowledge of the materials used by the artist. In recent years, in situ, noninvasive spectroscopic techniques have played an increasingly important role in art conservation. It has been extensively demonstrated that the synergic use of different spectroscopic methods helps to gain a deep understanding of the material composition of art objects while fully respecting their integrity and value. Moreover, the imaging and mapping systems (e.g., X-ray fluorescence scanner and hyperspectral cameras) play a relevant role as they give information about the materials distributed on large areas of studied samples.

To identify and characterize the different pictorial materials, among the many analytical techniques, diffuse reflectance spectroscopy in the ultraviolet-visible-near-infrared (UV-Vis-NIR) spectral range, about 200-2500 nm [1-3], has proven to be a powerful one, when applied in both laboratory and in situ on real artworks. If accurate in situ studies of

\footnotetext{
a e-mail: adele.sassella@unimib.it (corresponding author)
} 
real artworks over a wide spectral range is an important tool for historians and restorers, who need rich amounts of reliable data, it is mandatory to develop in laboratory experimental procedures on "known" samples to build databases to refer to.

In modern artworks, it is common to find acrylic colors which binders are hard to be precisely identified, because different acrylic polymers contain the same functional groups, like $-\mathrm{OH},-\mathrm{CH},-\mathrm{C}=\mathrm{O}$, and $-\mathrm{CH}_{3}$, and therefore, they have similar NIR spectra. For these reasons, literature papers about diffuse reflectance spectroscopy applied to works of art are principally aimed at understanding the characteristic response of pigments in the UV-Vis and the one of the binder in the NIR range. However, it has been recently demonstrated that in the NIR region the response of the polymeric binder can sometimes cover characteristic peaks of the pigments [4], thus impeding their precise identification. This may be a particularly relevant problem when the pigments are unrecognizable just by their UV-Vis spectra and other techniques are not conclusive; a typical example is the study of green compounds, mainly copper-based, which give similar results when analyzed by elemental analysis and UV-Vis spectroscopy, independently of the pigment composition [5-7]. To address the problem of separation and analysis of composite materials, including acrylic colors, some experimental techniques requiring complex experimental setup and procedures are well assessed in the literature (size exclusion chromatography-Fourier transform infrared spectroscopy SEC-FTIR, pyrolysis-gas chromatography-mass spectrometry Py-GC-MS) [8], while easier and simpler methods are still lacking.

In such a wide frame, the aim of this paper is twofold. First, it is aimed at developing a simpler method for the chemical separation of the components of acrylic colors, usually a mixture of binder, pigments, and fillers. A careful comparison between the spectroscopic response of the starting color obtained from various complementary techniques (such as UV-Vis-NIR diffuse reflectance, attenuated total reflection (ATR)-FTIR spectroscopy in mid-infrared (MIR) region, and Raman spectroscopy) and that of the extracted components is proposed to get full validation of the separation process. The second goal is demonstrating the possibility to build a database of reference spectra, to be used for future analysis and correction of the spectra collected in situ on real artworks. The proposed extraction method and spectroscopic analysis have been applied to original colors collected from tubes belonged to Domenico "Ico" Parisi (Palermo 1916-Como 1996), an Italian architect, designer, and painter.

\section{Experimental}

Two different acrylic colors used by Ico Parisi, found in his studio still within their original tubes and untouched since the $70 \mathrm{~s}$, have been studied. The first color is a "cobalt blue" made by Liquitex which contains C.I. Pigment Blue (PB) 28 (cobalt aluminate blue spinel $\mathrm{CoAl}_{2} \mathrm{O}_{4}$ ); the good preservation of the tube permits to recover some fluid color samples. The second color is a "cadmium red light" by Liquitex, containing C.I. Pigment Red (PR) 108 (cadmium selenosulfide CdS\CdSe); in this case, it was possible to extract just a bulk dry color sample. No indication about the exact composition of the binder is available for any of the colors in the tubes. Nonetheless, it is known [9] that Liquitex used aqueous acrylic emulsions with principally two different mixtures of polymers: in earlier times a blend of methyl methacrylate (MMA) and ethyl acrylate (EA), that polymerize in $\mathrm{p}(\mathrm{EA} \backslash \mathrm{MMA})$, and later a blend of MMA and n-butyl acrylate (nBA), that polymerize in $\mathrm{p}(\mathrm{nBA} \backslash \mathrm{MMA})$. 
For comparison, commercial modern pigments in powder form with the same C.I. generic name (PB28 for "blu cobalto," bought from CTS Srl, and PR108 for "rosso cadmio chiaro," bought from Zecchi Colori-Belle Arti di M e S Zecchi SNC) were analyzed.

For the spectroscopic studies of the different colors and pigments, thin solid films were prepared. From Parisi's cobalt blue sample, thin films are deposited by dripping a drop of the fluid color on a glass slide and then compressing it with a second glass slide, which was afterward scrolled away. Parisi's cadmium red light sample, being a bulk dry sample, was studied as it was. To form films of the commercial pure pigments, a small amount of powder (about $10 \mathrm{mg}$ ) was put into a vial with $5 \mathrm{ml}$ of tetrahydrofuran (THF), purchased from Fisher Scientific with GPC grade, and then sonicated to obtain a homogeneous suspension, which was then dripped on a glass slide; after solvent evaporation, a solid film of the pigment was obtained.

A chemical process was then designed and applied to both Parisi's samples to extract the pure pigment. First, a small amount of the original color from the tubes was put into a glass vial with $10 \mathrm{ml}$ of THF in order to swell and then disaggregate the polymer matrix; this step was made more efficient by putting the vial into an ultrasonic cleaner for $30 \mathrm{~min}$ with the heater on (at about $45^{\circ} \mathrm{C}$ ). The vial was then put into a centrifuge for $20 \mathrm{~min}$ at $3500 \mathrm{rpm}$, and the supernatant, resulting in a transparent and clear solution, was analyzed by ATR-FTIR spectroscopy to verify its composition. This entire process was repeated at least three times until the supernatant consisted of just THF, without any polymer residues (within the sensitivity limit of the ATR-FTIR instrument). To further verify the effectiveness of the treatment, ATR-FTIR measurements were carried out on the precipitated powder, left dry in air after centrifugation, supposed to be made of pigments only, to be compared with the ATR-FTIR spectra of the original color samples.

To prepare films for spectroscopic measurements, the pure pigment obtained after chemical separation was dispersed in $5 \mathrm{ml}$ of THF and sonicated to obtain a homogeneous suspension; the films were then prepared by drop casting on a glass slide, with the possibility of tuning film thickness by repeated drop depositions.

All the diffuse reflectance spectra were collected in the UV-Vis-NIR range (300-2500 nm) with $1 \mathrm{~nm}$ step by using a Perkin Elmer Lambda 900 spectrometer, equipped with a $15 \mathrm{~cm}$ integrating sphere; for the dry bulk sample, a homemade sample holder was used, which permitted the proper collection of the total reflected light (diffuse + specular), full reproducibility of the measurements, and correction of the background.

All the ATR-FTIR spectra were collected in the MIR range $\left(600-4000 \mathrm{~cm}^{-1}\right)$ with $1 \mathrm{~cm}^{-1}$ resolution and averaging 8 scans, using a Fourier Transform Perkin Elmer Spectrum 100 instrument, equipped with a diamond crystal, a KRS5 beam splitter, and a DTGS detector.

Raman spectra were collected on all the samples by a portable B\&W Tech I-Raman plus spectrometer, with fiber optic configuration, equipped with a microscope, with $785 \mathrm{~nm}$ excitation wavelength and a maximum output power of $500 \mathrm{~mW}$, and a resolution of $3 \mathrm{~cm}^{-1}$. In addition, to circumvent the problem [10] of the photoluminescence signal covering the Raman spectrum of some samples, a Jobin-Yvon Labram micro-Raman spectrometer equipped with a microscope was used for the extracted red cadmium (low energy region) and for the modern blue pigment; the excitation source was an internal He-Ne laser at $633 \mathrm{~nm}$, with maximum power $20 \mathrm{~mW}$, and Raman spectra were collected in back-scattering configuration through a CCD (charge coupled device) detector and corrected for spectral response of the apparatus. 


\section{Results and discussion}

The diffuse reflectance spectra of the two Parisi's color samples (Fig. 1a, b) show the characteristic response of the relative pigments in the Vis region $[11,12]$, while the bands detected in the NIR region could be attributed to the polymer matrix.

The polymer response in the NIR region is particularly evident in the cadmium red light spectrum, at 1180, 1435, 1700, 1924, and $2135 \mathrm{~nm}$ [13] (minima in the diffuse reflectance spectrum, corresponding to absorption maxima), related to the second overtones of strong chemical bonds like $-\mathrm{CH}$ and $-\mathrm{OH}$, while in the cobalt blue spectrum a characteristic response of the pigment from 950 to $1800 \mathrm{~nm}$ covers every other peak due to the polymer, except the one at $1930 \mathrm{~nm}$.

To verify the effectiveness of the separation method, the ATR-FTIR spectra of the samples obtained after chemical separation are compared to those collected on the original color samples from Parisi's tubes, i.e., still with all the components (Fig. 2a, b). The results clearly show that, for both color samples, most of the polymer is removed. Indeed, the characteristic sharp peak at $1730 \mathrm{~cm}^{-1}$ present in the two spectra of Parisi's colors and attributed to the stretching mode of the $-\mathrm{C}=\mathrm{O}$ bond of the acrylic group [14] is dominant in the spectrum before chemical separation. On the contrary, it is much less intense after the chemical process, as shown in Fig. 2a, where the peak at $1730 \mathrm{~cm}^{-1}$ has been used for normalizing the spectra. The other peaks remaining after treatment are therefore to be attributed to pigments and fillers.

Afterward, to verify that the pigment did not undergo chemical and/or chromatic changes during the chemical separation, diffuse reflectance is measured in the UV-Vis-NIR range on the two films of extracted pigment (Fig. 2c, d); in both cases the extracted pigment maintains the original characteristics, as the peculiar features in the spectra match those in the spectra of the untreated Parisi's colors. Looking at the pigment spectra, then, the cobalt blue has a wide absorption band from 950 to $1800 \mathrm{~nm}$, an absorption peak with maximum at $570 \mathrm{~nm}$ and another less intense at $475 \mathrm{~nm}$; the cadmium red absorption shows the cutting edge, typical of every red pigment [15]. This is therefore full experimental evidence that the peaks present in the NIR region of the original "cadmium red" color, as well as the peak at $1930 \mathrm{~nm}$ in the spectrum of the original "cobalt blue" color, are due only to the polymer matrix.

As a further check of the effectiveness of the separation method, the pigments extracted from Parisi's colors and the films of the corresponding commercial pigments are analyzed in terms of diffuse reflectance and ATR-FTIR spectra and compared.
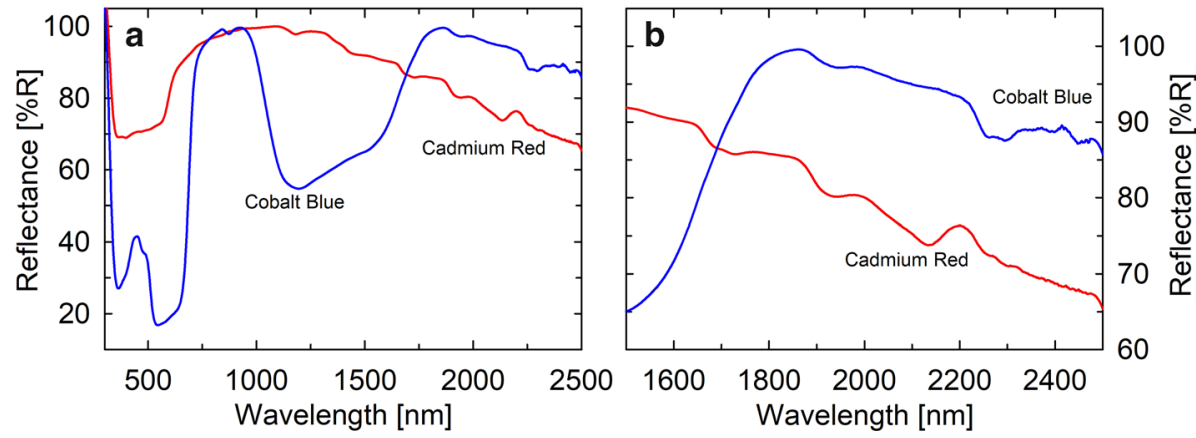

Fig. 1 a Diffuse reflectance spectra of cadmium red light (red curve) and cobalt blue (blue curve) colors from Parisi. b Zoom of the reflectance spectra in (a) in the NIR region 

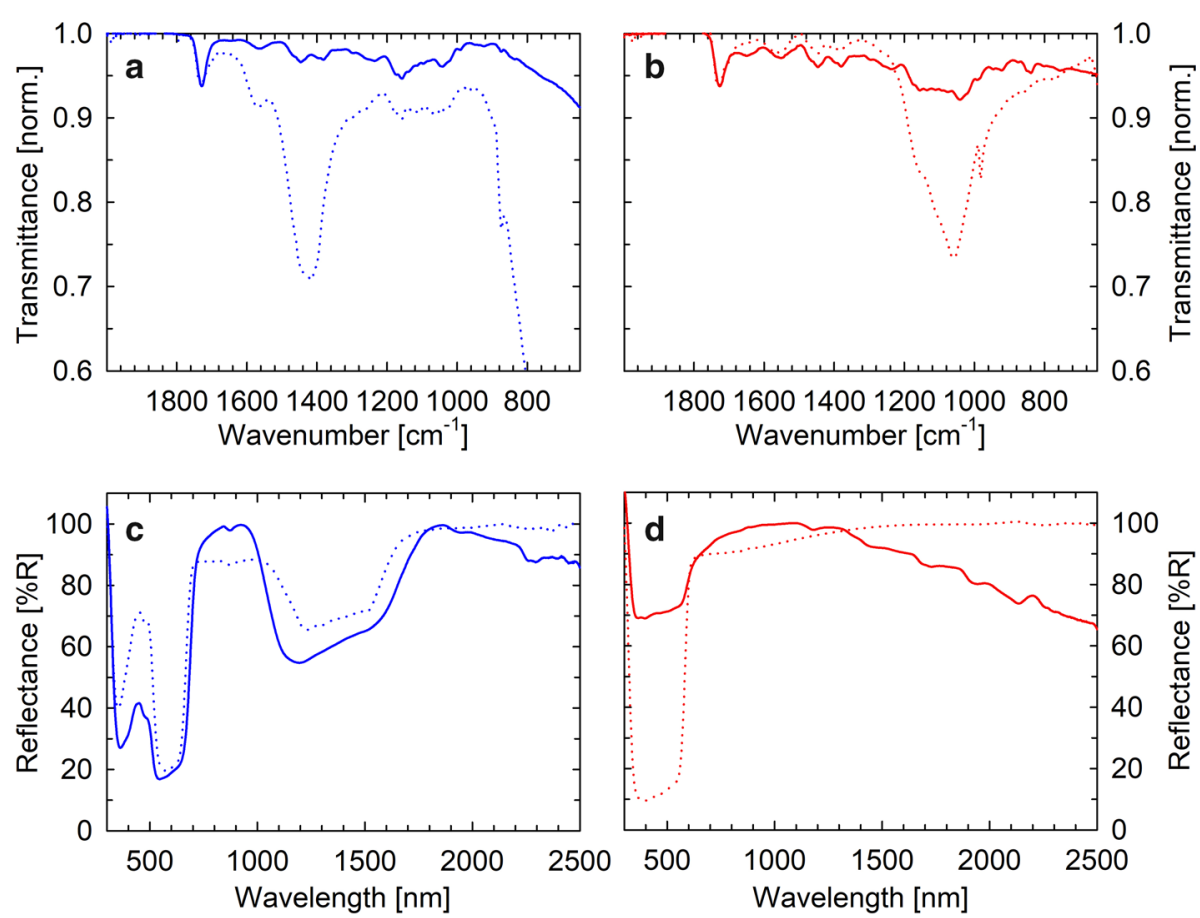

Fig. 2 a ATR-FTIR spectra of cobalt blue original sample (solid curve) and of the same sample after chemical separation (dotted curve); the two spectra are normalized to the peak assigned to the polymer matrix at $1730 \mathrm{~cm}^{-1}$. b ATR-FTIR spectra of cadmium red light original sample (solid curve) and of the same sample after chemical separation (dotted curve); the two spectra are normalized to the peak assigned to the polymer matrix at $1730 \mathrm{~cm}^{-1}$. c Diffuse reflectance spectra of cobalt blue original sample (solid curve) and of the same sample after chemical separation (dotted curve). d Diffuse reflectance spectra of cadmium red light original sample (solid curve) and of the same sample after chemical separation (dotted curve)

The diffuse reflectance spectra of the modern cobalt blue and the one extracted from Parisi's tube (Fig. 3a) clearly show that the samples contain the same pigment. Nonetheless, slight differences are observed: in particular, a steeper and slightly red-shifted increase of absorption at $720 \mathrm{~nm}$ and the presence of a small peak at $475 \mathrm{~nm}$ in the extracted pigment. Both samples present the same wide band in the NIR region and a maximum in absorption at $570 \mathrm{~nm}$. The commercial and extracted samples of cadmium red show the same spectra, with just a slight shift of about $10 \mathrm{~nm}$ in the absorption edge (Fig. 3b), and an almost flat long wavelength response, not exactly superimposed.

It should be noted that the ATR-FTIR spectra show some differences: both spectra (Fig. 3c, d) suggest that, in addition to the chromophore, pigments extracted from Parisi's colors and commercial pigments also contain fillers (not the same for both colors), masking completely the expected ATR-FTIR response of the pure chromophore. Such fillers can nonetheless be identified: the peaks at 1420,874 , and $713 \mathrm{~cm}^{-1}$ can be attributed to calcite [16] in the modern cadmium red spectrum and in the two samples of cobalt blue (extracted and commercial), while barium sulfate is responsible for the peaks at 1063 and $981 \mathrm{~cm}^{-1}$ [17], present in the spectrum of cadmium red from Parisi. Another filler is clearly present in the commercial cobalt blue, responsible for the two peaks at 1003 and $1086 \mathrm{~cm}^{-1}$; considering the spectral position of these MIR peaks, it can be supposed as a material containing $\mathrm{O}-\mathrm{Si}-\mathrm{O}$ groups, 

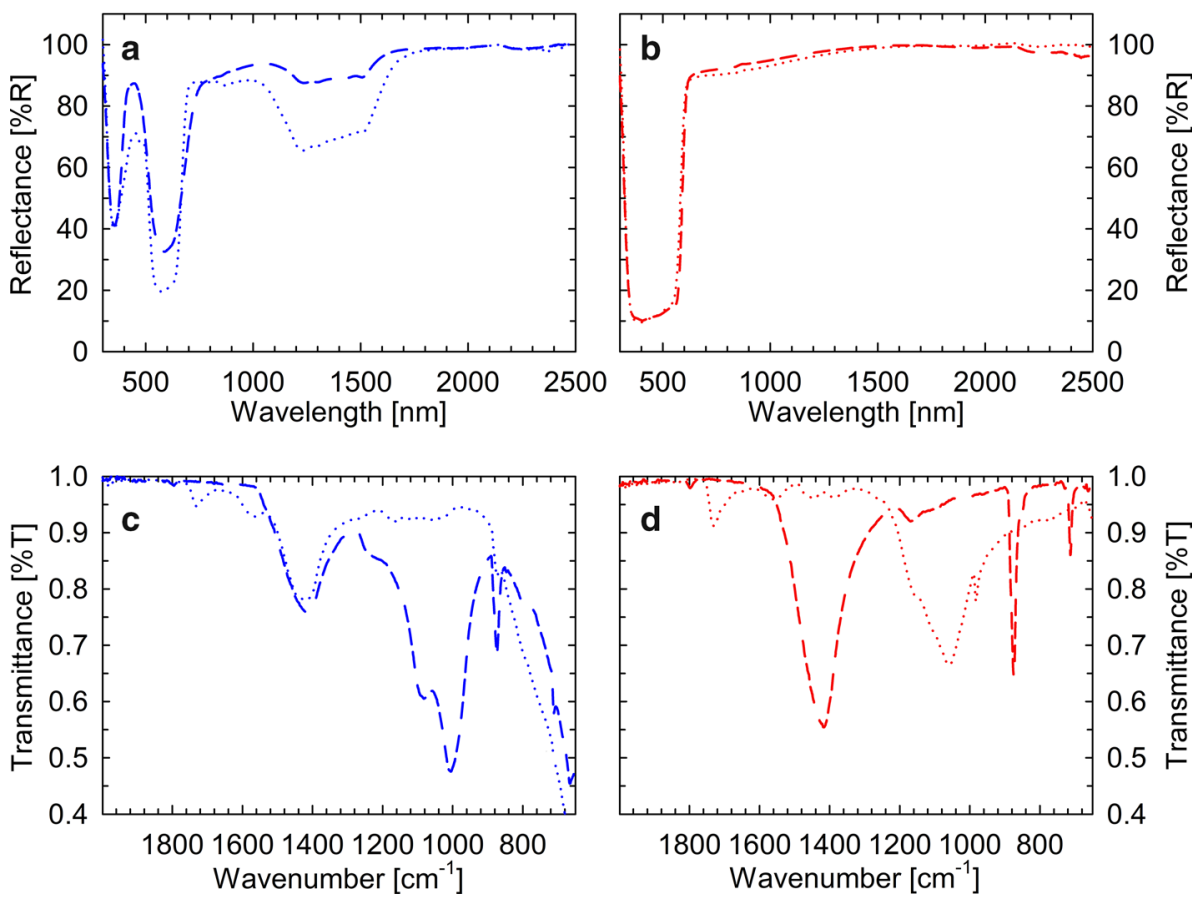

Fig. 3 a Diffuse reflectance spectra of cobalt blue extracted by chemical separation (dotted curve) and modern (dashed curve). b Diffuse reflectance spectra of cadmium red light extracted by chemical separation (dotted curve) and of modern pigment (dashed curve). c ATR-FTIR spectra of cobalt blue extracted by chemical separation (dotted curve) and modern (dashed curve). d ATR-FTIR spectra of cadmium red light extracted by chemical separation (dotted curve) and modern (dashed curve)

such as any clay materials [18]; a precise identification is prevented by the huge amount of clay materials and mixtures available and indeed commonly used as fillers [19, 20].

Back to the comparison between extracted pigments and their commercial counterparts, the presence of fillers does not allow to get a conclusive answer from the ATR-FTIR spectra; therefore, to definitively confirm the successful extraction of the pigments from Parisi's colors, a complementary technique, such as Raman spectroscopy, is applied to all the samples.

The spectra of cobalt blue samples are reported in Fig. $4 \mathrm{a}$, as acquired on the film of extracted pigment (dotted curve) and of the commercial one (dashed curve). The Raman spectrum of the extracted pigment confirms the results obtained by ATR-FTIR and diffuse reflectance measurements; in particular, the peaks at 200,408, and $511 \mathrm{~cm}^{-1}$ are due to the cobalt blue pigment $[21,22]$, while calcite, identified as filler, is responsible for the peaks at 276,708 , and $1085 \mathrm{~cm}^{-1}[21,23]$. The interpretation of the spectrum of the commercial pigment is tricky. Although the ATR-FTIR and reflectance spectra clearly demonstrate the presence of cobalt aluminate blue spinel $\mathrm{CoAl}_{2} \mathrm{O}_{4}$ as chromophore and calcite as filler, in the Raman spectrum only a barely visible peak at $200 \mathrm{~cm}^{-1}$ can be assigned to the chromophore (and the peak at $1090 \mathrm{~cm}^{-1}$ to calcite), nonetheless enough for full demonstration. Other peaks, ascribable to the clay materials already individuated by ATR-FTIR as possible fillers, are dominating the Raman spectrum.

Finally, the Raman spectrum of the red pigment extracted from Parisi's color is reported in Fig. 4b; it shows four peaks at 202, 293, 488, and $580 \mathrm{~cm}^{-1}$, all of them attributable 

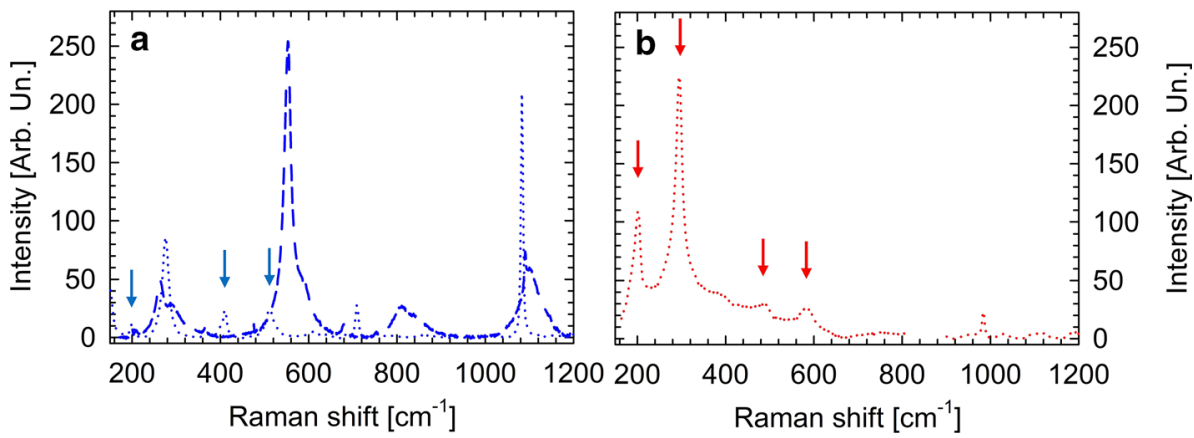

Fig. 4 a Raman spectra of the extracted blue (dotted line) and the modern blue pigment (dashed line). b Raman spectra of the extracted red. A 785-nm laser is used as excitation source for the extracted blue in (a) and for the high energy range (900-1200 $\left.\mathrm{cm}^{-1}\right)$ in (b), while the spectra of the modern blue in (a) and the low energy range (160-800 $\mathrm{cm}^{-1}$ ) in (b) are recorded with a 633-nm laser to get rid of luminescence. In both figures $\mathrm{A}$ and $\mathrm{B}$, the arrows indicate the peaks attributed to the pigments

to cadmium selenosulfide [21]. The presence of the filler identified as barium sulfate is responsible for the peak at $985 \mathrm{~cm}^{-1}$, while other peaks of this compound [24], known to be present in this spectral range but with lower intensity, are probably covered by the signal of the pigment. Direct comparison with the commercial pigment is not possible because of a strong luminescence signal observed both with the $785 \mathrm{~nm}$ and the 633-nm lasers, possibly originated from unknown fillers, covering the Raman peaks.

\section{Conclusions}

A method is proposed to study and separate the different contributions to the optical spectra of acrylic colors originating from their components. Even if this requires a destructive chemical treatment of the color samples, the response of the components can be used to build a database which can serve as future reference for the analysis of spectra recorded in situ on real artworks. To show how the results can be directly applicable to real cases, the method is here applied to two different acrylic colors recovered directly from tubes belonged to Domenico "Ico" Parisi, namely cobalt blue and cadmium red light. The comparison between diffuse reflectance spectra and ATR-FTIR spectra recorded on the original samples and those recorded on the extracted pigments show that the method is a reliable tool for separating pigment and filler from the polymeric binder. Then, thanks to the proposed chemical treatment, the diffuse reflectance spectra in the spectral region up to $2500 \mathrm{~nm}$ allow us to identify the characteristic features of the pigment alone, particularly relevant for cobalt blue, where this would not be possible without "cleaning" the spectra from the binder signal.

As secondary result, particularly relevant for future studies, the comparison between the extracted pigments and their modern counterparts highlights the difficulty in finding modern colors with the exact same composition as those used in the past. In our case, on the one hand reflectance spectra show how the pigment itself could be slightly different, while, on the other hand, ATR-FTIR and Raman spectra enlighten how totally different fillers can be present in modern colors with respect to those used in the past.

Following our method, the original mixture of pigment and fillers can be identified, recovered, and reused, giving worthy results in the field of history studies and restoration. Finally, 
we plan to take advantage of the knowledge of Ico Parisi's palette to characterize his artworks directly at Fondazione Ico Parisi (Como, Italy) where they are exhibited.

Acknowledgements This work was supported by Regione Lombardia (Italy) in the framework of the Project "MOBARTECH: una piattaforma mobile tecnologica, interattiva e partecipata per lo studio, la conservazione e la valorizzazione di beni storico-artistici-Call Accordi per la Ricerca e l'Innovazione." The authors gratefully acknowledge Dr. Roberto Lorenzi for his kind support with some of the Raman measurements and Prof. Antonio Papagni for useful discussion.

Funding Open access funding provided by Università degli Studi di Milano - Bicocca within the CRUI-CARE Agreement.

\section{Compliance with ethical standards}

Conflict of interest The authors declare no conflict of interest.

Open Access This article is licensed under a Creative Commons Attribution 4.0 International License, which permits use, sharing, adaptation, distribution and reproduction in any medium or format, as long as you give appropriate credit to the original author(s) and the source, provide a link to the Creative Commons licence, and indicate if changes were made. The images or other third party material in this article are included in the article's Creative Commons licence, unless indicated otherwise in a credit line to the material. If material is not included in the article's Creative Commons licence and your intended use is not permitted by statutory regulation or exceeds the permitted use, you will need to obtain permission directly from the copyright holder. To view a copy of this licence, visit http://creativecommons.org/licenses/by/4.0/.

\section{References}

1. M. Aceto, A. Agostino, G. Fenoglio, A. Idone, M. Gulmini, M. Picollo, P. Ricciardi, J.K. Delaney, Anal. Methods 6, 1488 (2014)

2. J. Striova, A. Dal Fovo, R. Fontana, Riv. Nuovo Cim. 43, 515 (2020)

3. N. Odisio, M. Calabrese, A. Idone, N. Seris, L. Apollonia, J.M. Christille, Eur. Phys. J. Plus 134, 67 (2019)

4. R. Yivlialin, A. Galli, L. Raimondo, M. Martini, A. Sassella, Heritage 2, 2255 (2019)

5. E. Platania, M.L.W. Streeton, A. Lluveras-Tenorio, A. Vila, D. Buti, F. Caruso, H. Kutzke, A. Karlsson, M.P. Colombini, E. Uggerud, Microchem. J. 156, 1 (2020)

6. J. Buse, V. Otero, M. Melo, Heritage 2, 1614 (2019)

7. P. Ricciardi, A. Pallipurath, K. Rose, Anal. Methods 5, 3819 (2013)

8. D. Scalarone, O. Chiantore, J. Sep. Sci. 27, 263 (2004)

9. J.H. Stoner, R. Rushfield, in Modern Paints. ed. by A. Oddy (Routledge, London, 2012), p. 242

10. D. Wei, S. Chen, Q. Liu, Appl. Spectrosc. Rev. 50, 387 (2015)

11. M. Thoury, J.K. Delaney, E.R. De la Rie, M. Palmer, K. Morales, J. Krueger, Appl. Spectrosc. 65, 939 (2011)

12. M. Bacci, M. Picollo, Stud. Conserv. 41, 136 (1996)

13. J. Workman Jr., Handbook of Organic Compounds. NIR, IR, Raman, and UV-Vis Spectra Featuring Polymers and Surfactants (Academic Press, Cambridge, 2001).

14. A.H. Kuptsov, G.N. Zhizhin, Handbook of Fourier Transform Raman and Infrared Spectra of Polymers (Elsevier, Amsterdam, 1998).

15. E. West Fitzhugh, Red Lead and Minium. ed. by R.L. Feller (Washington Archetype Publications, London, 1986), p. 109

16. G.C. Jones, B. Jackson, Infrared Transmission Spectra of Carbonate Minerals (Chapman \& Hall, London, 1993).

17. F. Jones, Cryst. Eng. Comm. 14, 8374 (2012)

18. J. Madejovà, Vib. Spectrosc. 31, 1 (2003)

19. W. Roelle, in Extenders. ed. by D. Stoye, W. Freitag (Wiley-CCH, New York, 1998), p. 150 
20. H.H. Murray, Appl. Clay Sci. 17, 207 (2000)

21. M.C. Caggiani, A. Cosentino, A. Mangone, Microchem. J. 129, 123 (2016)

22. M. Bouchard, A. Gambardella, J. Wiley, J. Raman. Spectrosc. 41, 1477 (2010)

23. H.N. Rutt, J.H. Nicola, J. Phys. C Solid State Phys. 7, 4522 (1974)

24. N.V. Chukanov, M.F. Vigasina, Vibrational (Infrared and Raman) Spectra of Minerals and Related Compounds (Springer, Cham, 2020). 